\title{
The crystal structure of 2-bromoisophthalic acid, $\mathrm{C}_{8} \mathrm{H}_{5} \mathrm{BrO}_{4}$
}

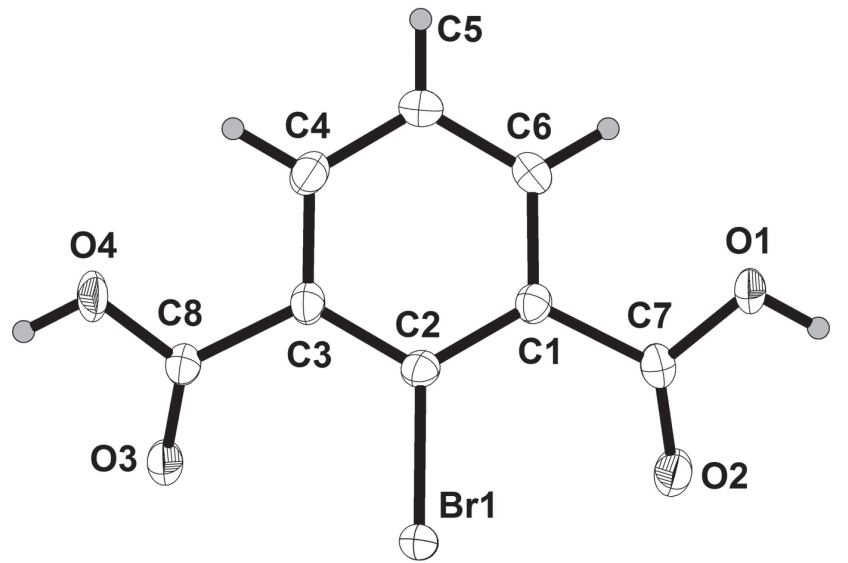

https://doi.org/10.1515/ncrs-2019-0457

Received June 30, 2019; accepted July 25, 2019; available online September 11, 2019

\begin{abstract}
$\mathrm{C}_{8} \mathrm{H}_{5} \mathrm{BrO}_{4}, \quad$ triclinic, $\quad P \overline{1} \quad$ (no. 2), $\quad a=4.8161$ (3) $\AA$, $b=7.3727(5) \AA, \quad c=12.1230(7) \AA, \quad \alpha=81.004(3)^{\circ}$, $\beta=78.897(3)^{\circ}, \quad \gamma=72.829(3)^{\circ}, \quad V=401.30(4) \AA^{3}, \quad Z=2$, $R_{\mathrm{gt}}(F)=0.0201, w R_{\text {ref }}\left(F^{2}\right)=0.0479, T=150(2) \mathrm{K}$.
\end{abstract}

\section{CCDC no.: 1941305}

The crystal structure is shown in the figure. Table 1 contains crystallographic data and Table 2 contains the list of the atoms including atomic coordinates and displacement parameters.

\section{Source of materials}

All of reagents are commercial resource and used as received. The title organic molecule was recrystallized as follows: under stirring, to a THF solution (10 mL), $1 \mathrm{mmol} \mathrm{2-}$ bromoisophthalic acid ( $0.245 \mathrm{~g}$ ) was added and heated at $50{ }^{\circ} \mathrm{C}$ for $1 \mathrm{~h}$. The filtrate let evaporate in air at room temperature. A large number of colorless crystals were obtained after several days, washed with a small amount of water and air-dried.

\footnotetext{
*Corresponding author: Shu-Jing Li, Department of Chemistry and Chemical Engineering, Zhoukou Normal University, Zhoukou, Henan Province 466001, P. R. China, e-mail: zksylsj@163.com

Ke Li and Yi-Wen Li: Department of Chemistry and Chemical Engineering, Zhoukou Normal University, Zhoukou, Henan Province 466001, P. R. China
}

Ә Open Access. ( 2019 Shu-Jing Li et al., published by De Gruyter. (c) BY License.
Table 1: Data collection and handling.

\begin{tabular}{ll}
\hline Crystal: & Colorless block \\
Size: & $0.3 \times 0.25 \times 0.1 \mathrm{~mm}$ \\
Wavelength: & Mo $K \alpha$ radiation $(0.71073 \AA$ A $)$ \\
$\mu:$ & $5.10 \mathrm{~mm}^{-1}$ \\
Diffractometer, scan mode: & Bruker APEX-II, $\varphi$ and $\omega$-scans \\
$\theta_{\text {max }}$, completeness: & $26.5^{\circ},>99 \%$ \\
$N(h k l)_{\text {measured }}, N(h k l)_{\text {unique }}, R_{\text {int }}:$ & $8569,1646,0.034$ \\
Criterion for $I_{\text {obs }}, N(h k l)_{\text {gt }}:$ & $I_{\text {obs }}>2 \sigma\left(I_{\text {obs }}\right), 1490$ \\
$N(\text { param })_{\text {refined }}:$ & 120 \\
Programs: & Bruker programs [1], SHELX [2], \\
& OLEX2 [3, 4] \\
\hline
\end{tabular}

Table 2: Fractional atomic coordinates and isotropic or equivalent isotropic displacement parameters $\left(\AA^{2}\right)$.

\begin{tabular}{lrrrr}
\hline Atom & $\boldsymbol{x}$ & $\boldsymbol{y}$ & $\boldsymbol{z}$ & $\boldsymbol{U}_{\text {iso }}{ }^{*} \boldsymbol{U}_{\text {eq }}$ \\
\hline Br1 & $0.76797(5)$ & $0.08612(3)$ & $0.823044(18)$ & $0.02095(8)$ \\
C1 & $0.5975(4)$ & $0.4959(3)$ & $0.79963(16)$ & $0.0114(4)$ \\
C2 & $0.5992(4)$ & $0.3320(3)$ & $0.75373(16)$ & $0.0117(4)$ \\
C3 & $0.4436(4)$ & $0.3504(3)$ & $0.66412(17)$ & $0.0125(4)$ \\
C4 & $0.2717(4)$ & $0.5296(3)$ & $0.62740(17)$ & $0.0145(4)$ \\
H4 & $0.1610(4)$ & $0.5414(3)$ & $0.56848(17)$ & $0.0174(5)^{\star}$ \\
C5 & $0.2597(5)$ & $0.6911(3)$ & $0.67535(18)$ & $0.0162(4)$ \\
H5 & $0.1381(5)$ & $0.8121(3)$ & $0.65085(18)$ & $0.0194(5)^{\star}$ \\
C6 & $0.4265(5)$ & $0.6740(3)$ & $0.75926(17)$ & $0.0148(4)$ \\
H6 & $0.4250(5)$ & $0.7852(3)$ & $0.79004(17)$ & $0.0178(5)^{\star}$ \\
C7 & $0.7764(4)$ & $0.4919(3)$ & $0.88843(17)$ & $0.0128(4)$ \\
C8 & $0.4642(5)$ & $0.1883(3)$ & $0.59985(17)$ & $0.0133(4)$ \\
O1 & $0.6806(3)$ & $0.6476(2)$ & $0.94095(13)$ & $0.0187(3)$ \\
H1 & $0.792(3)$ & $0.6424(18)$ & $0.9876(17)$ & $0.0280(5)^{\star}$ \\
O2 & $0.9923(3)$ & $0.3629(2)$ & $0.90761(13)$ & $0.0185(3)$ \\
O3 & $0.6813(3)$ & $0.0537(2)$ & $0.58514(13)$ & $0.0187(3)$ \\
04 & $0.2260(3)$ & $0.2132(2)$ & $0.55388(13)$ & $0.0197(3)$ \\
H4a & $0.251(2)$ & $0.123(2)$ & $0.5151(19)$ & $0.0295(5)^{\star}$ \\
\hline
\end{tabular}

\section{Experimental details}

All $\mathrm{H}$-atoms from $\mathrm{C}$ atoms and $\mathrm{O}_{\text {hydroxyl }}$ atoms were positioned with idealized geometry and refined isotropically $\left(U_{\text {iso }}(\mathrm{H})=1.2 U_{\text {eq }}(\mathrm{C})\right.$ or $1.5 U_{\text {eq }}(\mathrm{O})$ for all $\mathrm{H}$ atoms $)$ using a riding model with $\mathrm{C}-\mathrm{H}=0.95 \AA$ and $\mathrm{O}-\mathrm{H}=0.84 \AA$.

\section{Comment}

Several crystal structures of bromine-substituted isophthalic acids or their derivatives have been reported, 
such as 5-bromoisophthalic acid [5, 6], 5-amino-2,4,6tribromoisophthalic acid [7], 4,6-dibromoisophthalic acid monohydrate [8], 4,4'-dibromo-1,1'-biphenyl-2,2',6,6'tetracarboxylic acid [9], 2-bromobenzene-1,3,5-tricarboxylic acid monohydrate [10], 2,5-dibromoisophthalic acid monohydrate and 5-bromo-2-((2-methoxyphenyl)(phenyl)amino) isophthalic acid [11]. However, the crystal structure of 2-bromoisophthalic acid has not been reported anywhere. Thus, we herein report the crystal structure of the title compound.

As shown in the figure, the title compound, named 2-bromoisophthalic acid, with the molecule formula $\mathrm{C}_{8} \mathrm{H}_{5} \mathrm{BrO}_{4}$, crystalizes in the triclinic space group $P \overline{1}$. All bond lengths and angles of the title compound are normal, compared with its analogues [5-11]. The benzene ring is nearly planar. The two carboxyl groups are not coplanar with the aforementioned ring. There are two kinds of $\mathrm{O}-\mathrm{H} \cdots \mathrm{O}$ hydrogen bonds: one is $\mathrm{O} 1-\mathrm{H} 1 \cdots \mathrm{O} 2$, the other is $04-\mathrm{H} 4 \mathrm{a} \cdot \mathrm{O} 3$ 3, with their D.A A distances 2.6183(24) and 2.6722(23) A, respectively. These hydrogen bonds link molecules to generate a one-dimensional chain.

Acknowledgements: This study was supported by Pei Ying Plan of SIVA (Z.30001.16.05.16.14) and Education Department of Highland Construction Action Plan of SIVA (Z.31008.17.06). Dr. G. Huang also thanks Chinese Academy of Cultural Heritage for his postdoctoral position.

\section{References}

1. Bruker. SAINT v8.37A. Bruker AXS Inc., Madison, WI, USA (2015).
2. Sheldrick, G. M.: A short history of SHELX. Acta Crystallogr. A64 (2008) 112-122.

3. Bourhis, L. J.; Dolomanov, O. V.; Gildea, R. J.; Howard, J. A. K.; Puschmann, H.: The anatomy of a comprehensive constrained, restrained refinement program for the modern computing environment-Olex2 dissected. Acta Crystallogr. A71 (2015) 59-75.

4. Dolomanov, O. V.; Bourhis, L. J.; Gildea, R. J.; Howard, J. A. K.; Puschmann, H.: OLEX2: a complete structure solution, refinement and analysis program. J. Appl. Crystallogr. 42 (2009) 339-341.

5. Therrien, B.; Vieille-Petit, L.; Suss-Fink, G.: X-ray crystallographic study of hydrogen-bonded systems formed between di- and tricarboxylic acids and the trinuclear cluster cation $\left[\mathrm{H}_{3} \mathrm{Ru}_{3}\left(\mathrm{C}_{6} \mathrm{H}_{6}\right)\left(\mathrm{C}_{6} \mathrm{Me}_{6}\right)_{2}(0)\right]^{+}$. J. Mol. Struct. 749 (2005) 183-186.

6. Dan Li, D.; Yang, X.; Yan, D.: Cluster-based metal-organic frameworks: modulated singlet-triplet excited states and temperature-responsive phosphorescent switch. ACS Appl. Mater. Interfaces. 10 (2018) 34377-34384.

7. Beck, T.; Herbst-Irmer, R.; Sheldrick, G. M.: 5-Amino-2,4,6tribromo-isophthalic acid: the MAD triangle for experimental phasing. Acta Crystallogr. C65 (2009) 0237-0239.

8. Ye, B.: 4,6-Dibromo-isophthalic acid monohydrate. Acta Crystallogr. E68 (2012) 02642.

9. Holya, P.; Zavada, J.; Zezulaa, J.; Cisarova, I.; Jaroslav Podlahab, J.: Formation of infinite grids with chiral square compartments by self-assembly of achiral $1,1^{\prime}$-biphenyl-2,2',6, $6^{\prime}$ tetracarboxylic acids. Effect of 4,4'-substitution von the grid stacking. Collect. Czech. Chem. Commun. 66 (2001) 820-832.

10. Munch, A. S.; Katzsch, F.; Gruberb, T.; Mertensa, F. O. R. L.: Spectroscopic, thermal and single crystal structure investigations of 2-bromotrimesic acid and its trimethyl ester analogue. J. Mol. Struct. 1074 (2014) 542-548.

11. Surampudi, S. K.; Nagarjuna, G.; Okamoto, D.; Chaudhuri, P. D.; Venkataraman, D.: Apical functionalization of chiral heterohelicenes. J. Org. Chem. 77 (2012) 2074-2079. 